\title{
Variation of Glycosylated Hemoglobin (HbA1C) Cutoff among type-2 diabetic nephropathy Patients
}

\author{
Mohammed Idrees Khan ${ }^{1 *}$, Fauzia Ashfaq ${ }^{2}$ \\ ${ }^{1}$ Assistant Professor, Department of Clinical Nutrition, College of Applied Health Sciences in Arras, Qassim University \\ ${ }^{2}$ Assistant Professor,Department of Clinical Nutrition,Abu Arish Community College, Jazan University,Jazan(K.S.A)
}

Received: August 28,2018; Accepted: September 19,2018; Published: October 26,2018

*Corresponding author: Mohammed Idrees Khan, Assistant Professor, Department of Clinical Nutrition, College of Applied Health Sciences in Arras, Qassim University (K.S.A),Tel: +966-537234624,E-mail: mohdidreesh06@gmail.com

\section{Abstract}

Objectives: The aim of the study was to determine glycosylated hemogobin (HbA1c) cut-off and lipid correlation, duration of diabetes with level of proteinuria.

Materials And Methods: Cross sectional study was done on 250 patients with type- 2 diabetes mellitus subjects attending medicine clinic, King george's medical university (KGMU) lucknow, india. Subjects were screened for diabetic nephropathy depending on the base line parameter, clinical history of disease and documented in pretested proforma. Routine blood parameters, $\mathrm{HbA1C}$ and $24 \mathrm{hrs}$ urine microalbumin level were carried out in department of pathology.

Result: The level of HbA1c was higher among the patients of nephropathy $(8.54 \pm 0.97)$ compared with non-nephropathy $(7.32 \pm 0.84$, 95\%CI=7.17-7.47). Increased HbA1c was found among both 5-10 and $>10$ years diabetic duration . However, $\mathrm{HbA1} \mathrm{c}$ was similar among all duration of diabetes groups within non-nephropathy. The total cholesterol (TC), HDL cholesterol (HDL-c), LDL cholesterol (LDL-c) and VLDL cholesterol (VLDL-C) were almost similar in HbA1c range $<7,7-8.9$ and 9-10.9 and without insignificant differences ( $p>0.05$ ). Although, Triglyceride (TG) level was significantly higher in HbA1c 9-10.9 (174.20 $\pm 48.95 \mathrm{mg} \%)$ than 7-8.9 $(159.66 \pm 31.47 \mathrm{mg} \%)$ and $<7$ $(158.82 \pm 29.67 \mathrm{mg} \%)$. High prevalence of nephropathy was found among HbA1c range 9-10.9 (Urine albumin :246.35 $\pm 126.29 \mathrm{mg} / \mathrm{d}$ ) compared with 7-8.99 (Urine albumin : $159.89 \pm 70.16 \mathrm{mg} / \mathrm{d}$ ) and $<7$ (Urine albumin : $132.80 \pm 90.33 \mathrm{mg} / \mathrm{d}$ ) respectively.

Conclusion : HbA1c cut-off $>7 \%$ should be considered as an index of glycemic control as well as important tool for over all metabolic derangement and target organ damage among diabetes population. Hence, establishment of HbA1c cut-off value with long duration of diabetes might be useful for prediction of treatment control and prevention of renal failure.

Keywords; Diabetic Nephropathy; Glycosylated Hemoglobin; Triglyceride; Renal failure

\section{Introduction}

Diabetes Mellitus (DM) is a metabolic disorders associated with to micro- and macro-vascular complications. Microvascular complications include neuropathy, retinopathy, and nephropathy. Macrovascular complications are coronary artery and peripheral artery disease. In large major complication is linked with all major organ damage leads to morbidity and mortality.[1,2]
Diabetic nephropathy (DN) is leading cause of end stage renal disease (ESRD) associated with high rates of morbidity and mortality.[3] Early identification and treatment of nephropathy complication can reduce the medical and economic burden of major damage.[4] Although microalbuminuria is a widely used indicator for diabetic nephropathy, its diagnostic accuracy is limited by the fact that structural damage might precede albumin excretion.[5]

Numerous guidelines have defined HbA1c as marker of mean blood glucose levels and as a priority therapeutically.[6] Chronic hyperglycemia is responsible for the development of complications in diabetes patients. Furthermore, glucose variability could be a predictor of complications. Glucose variability could be defined in several ways: within-day variability, between-day variability and long-term variability expressed using changes in HbA1c. .[7] Diabetes Control and Complications Trial (DCCT) evidenced that $\mathrm{HbA1c}$ variability, similar to mean A1c levels, could predict the development of nephropathy and retinopathy in T1DM patients . [8] Intra-person standard deviation in HbA1C was an independent risk factor for the development of microalbuminuria in T2DM Tsukuba Kawai Diabetes Registry .[9]

Although numerous prevalence and incidence risk factors studies have been carried out to ruled out correlation for diabetic nephropathy but limitation of prognostic as well as biomarker of glycemic control was not emphasized properly in previous studies. Aim of this study to determine association of HbA1c cut-off variation in nephropathy complication with relation to duration of diabetes and dyslipidemia.

\section{Material \& Methods}

The cross sectional study designs were used for risk factors for nephropathy .The study has been carried out in type-2 diabetic mellitus subjects attending medicine clinic, KGMU , had been screened for nephropathy. Institutional Ethical Committee of KGMU granted ethical clearance for study in 2014. Diabetic in the age group of 40 - 69 years, with fixed OHA dosage and receiving angiotensin- converting enzyme inhibitors or angiotensin receptor blockers for screening of microalbuminuria were recruited. 
Diabetic subjects with other concurrent acute illness including infectious disease, malignancy, active immunological diseases, medical history of clinical cardiovascular disease, tuberculosis, pregnancy, lactation, using corticosteroids or other medicines such as statins, or vitamins, or mineral supplements in the past 3 months, severe uncontrolled hypertension (> 140/90 mmHg) or renal insufficiency (serum creatinine $>1.5 \mathrm{mg} / \mathrm{dl}$ ), who were receiving insulin preparations as a part of diabetes management, individuals with hematuria/pyuria/urinary tract infections/ ketonuria at the time of screening, who had performed strenuous physical exercise and smoking history were excluded from the study.

The risk of nephropathy among type-2 diabetes was being reported to be $15-20 \% .10$ (Vishwanathan et.al, 1998). Assuming $80 \%$ power and $5 \%$ significance level, the sample size was calculated by using the formula for risk factor objective, the calculated sample size was 246 . Therefore, total of 250 patients were enrolled in the study for risk factor of nephropathy.

Routine Blood Parameter and HbA1c was estimated in ethylenediaminetetraacetic acid anti coagulated whole blood by ion exchange chromatography. TC,TG, HDL-c were measured by enzymatic methods using Hitachi 917 auto analyzer with its original reagent. (Accurex Biochemical Pvt. Ltd, Mumbai, India).

$24 \mathrm{hrs}$ Urine albumin estimation was done after persistent proteinuria detection by dipstick methods a gap of one week and urine microscopic examination to rule out any infection, then 24-h quantitative determination of microalbumin in urine by turbid metric immunoassay based on antigen- antibody reaction in measurement by the end point method (Erba Diagnostics Mannheim GmbH, Mallaustrasse Mannheim / Germany.).

\section{Statistical analysis}

The data collected was entered in Microsoft Excel program and was checked for any inconsistency. A one - sample Kolmogorov Smirnov test was used to investigate whether the variables were normally distributed. The unpaired t- test was used to investigate the differences at the baseline values among two groups. The statistical significance was accepted at a probability level of less than 0.05 . Analyses were performed by using SPSS software package (WINDOWS version 15.0: SPSS Inc., Chicago, IL,USA)

\section{Result}

Mean age was almost similar among both groups. Long duration of diabetes $11.57 \pm 3.58$ years was found in nephropathy. Blood pressures were significantly $(\mathrm{p}<0.0001)$ higher among nephropathy (systolic: 133.52 \pm 6.81 , diastolic: $81.09 \pm 4.74$ mmhg) as compared to non-nephropathy ((systolic: 129.12 \pm 4.58 , diastolic: 77.24 \pm 7.36 ). Fasting blood glucose (FBG) was higher among nephropathy $(153.37 \pm 34.24)$ than non-nephropathy (123.54 \pm 51.78$)$ and post-prandial blood glucose (PPBG) similar difference was observed. (Table-1) The level of HbA1c was higher among nephropathy $(8.54 \pm 0.97,95 \% \mathrm{CI}=8.37-8.71)$ than nonnephropathy $(7.32 \pm 0.84,95 \% \mathrm{CI}=7 \cdot 17-7.47)$. (Fig:1).
Table 1: Comparison of baseline characteristics of Type-2 Diabetic subjects

\begin{tabular}{|c|c|c|c|}
\hline Characteristics & $\begin{array}{c}\text { Non- } \\
\text { nephropathy } \\
\text { (n=120) }\end{array}$ & $\begin{array}{c}\text { Nephropathy } \\
\text { (n=130) }\end{array}$ & p-value \\
\hline Age in years & $54.86 \pm 8.48$ & $57.37 \pm 6.21$ & 0.06 \\
\hline $\begin{array}{c}\text { Duration of } \\
\text { diabetes (years) }\end{array}$ & $7.22 \pm 1.83$ & $11.57 \pm 3.58$ & 0.001 \\
\hline BMI (kg/m $)$ & $24.76 \pm 2.22$ & $27.14 \pm 2.06$ & 0.0001 \\
\hline $\begin{array}{c}\text { Systolic blood } \\
\text { pressure (mmHg) }\end{array}$ & $129.12 \pm 4.58$ & $133.52 \pm 6.81$ & 0.0001 \\
\hline $\begin{array}{c}\text { Diastolic blood } \\
\text { pressure (mmHg) }\end{array}$ & $77.24 \pm 7.36$ & $81.09 \pm 4.74$ & 0.0001 \\
\hline Fasting blood sugar & $123.54 \pm 51.78$ & $153.37 \pm 34.24$ & 0.0001 \\
\hline $\begin{array}{c}\text { Post-prandial } \\
\text { blood sugar }\end{array}$ & $171.06 \pm 37.70$ & $208.09 \pm 51.38$ & 0.0001 \\
\hline HbA1c & $7.17 \pm 0.84$ & $8.37 \pm 0.97$ & 0.0001 \\
\hline Serum urea & $22.55 \pm 6.84$ & $30.10 \pm 6.56$ & 0.0001 \\
\hline Serum creatinine & $0.92 \pm 0.31$ & $1.14 \pm 0.28$ & 0.0001 \\
\hline
\end{tabular}

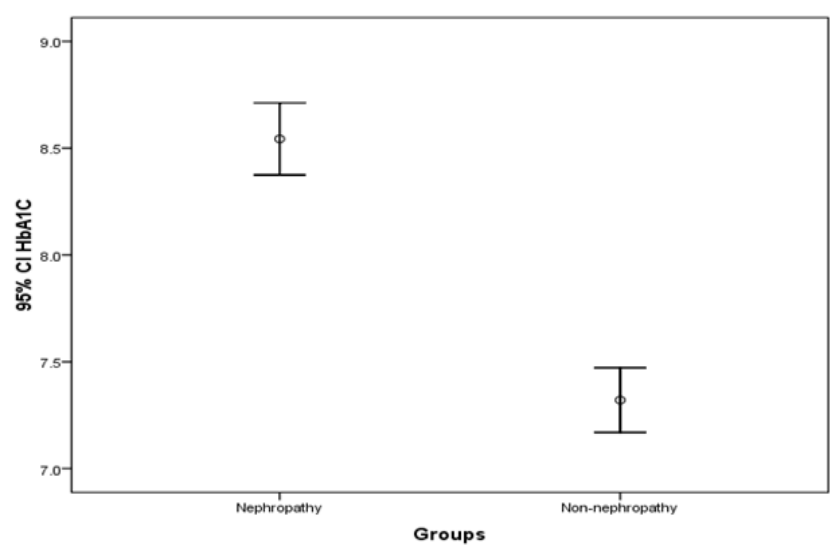

Figure 1: HbA1c level between nephropathy and non-nephropathy patients

The HbA1c was significantly $(\mathrm{p}=0.008)$ higher among nephropathy $(7.90 \pm 0.45)$ patients of duration $<5$ years than nonnephropathy $(7.17 \pm 0.75)$ patients. Similar observation was found for duration of diabetes of 5-10. The HbA1c was significantly $(p=0.03)$ different between duration of diabetes $<5$ vs $5-10$ $(\mathrm{p}=0.04)$ and $<5$ vs $>10 \quad(\mathrm{p}=0.02)$ years within nephropathy patients. However, HbA1c was similar among all duration of diabetes groups within non-nephropathy patients ( Fig 2).

TC, HDL-c, LDL-c and VLDL-c were almost similar in HbA1c <7, 7-8.9 and 9-10.9 without insignificant difference ( $p>0.05)$. However, TG level was significantly higher in HbA1c

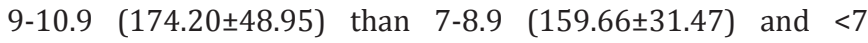
(158.82 \pm 29.67 ) (Table 2). 


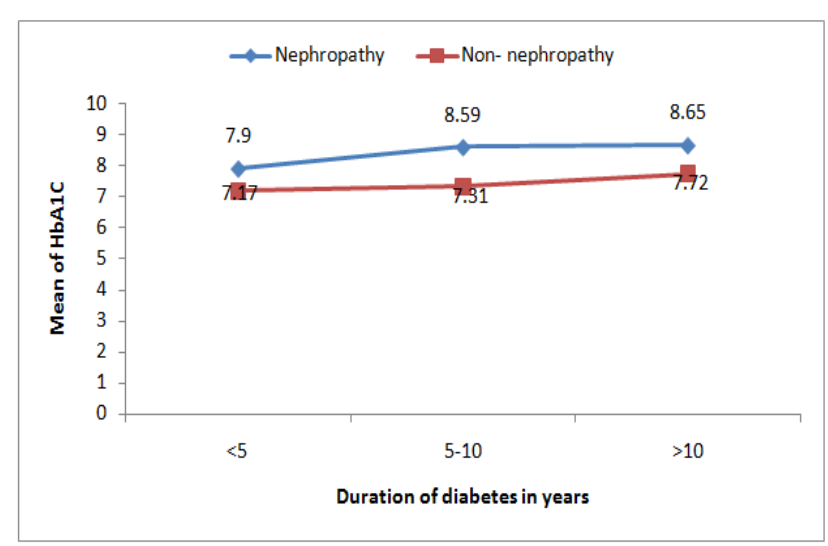

Figure 2: HbA1c level by duration of diabetes between nephropathy and non-nephropathy patients

\begin{tabular}{|c|c|c|c|c|}
\hline \multirow{2}{*}{$\begin{array}{c}\begin{array}{c}\text { Lipid } \\
\text { profile }\end{array} \\
\text { (mg/dl) }\end{array}$} & \multicolumn{3}{|c|}{ HbA1c \% } & \multirow[b]{2}{*}{ Significance } \\
\hline & $\begin{array}{c}<7 \\
(n=5)\end{array}$ & $\begin{array}{c}7-8.9 \\
(n=83)\end{array}$ & $\begin{array}{l}9-10.9 \\
(n=42)\end{array}$ & \\
\hline $\begin{array}{c}\text { Total } \\
\text { cholesterol }\end{array}$ & $151 \pm 30.94$ & $152.33 \pm 20.64$ & $156.16 \pm 25.83$ & $\mathrm{~F}=1.85, \mathrm{p}=0.17$ \\
\hline TG & $158.82 \pm 29.67$ & $159.66 \pm 31.47$ & $174.20 \pm 48.95$ & $\mathrm{~F}=5.60, \mathrm{p}=0.002^{*}$ \\
\hline HDL-c & $26.40 \pm 4.92$ & $25.78 \pm 7.27$ & $24.57 \pm 4.69$ & $\mathrm{~F}=0.35, \mathrm{p}=0.71$ \\
\hline LDL-c & $96.60 \pm 20.25$ & $97.28 \pm 19.43$ & $99.88 \pm 28.00$ & $F=1.15, p=0.85$ \\
\hline VLDL-c & $36.60 \pm 7.16$ & $37.89 \pm 6.33$ & $38.99 \pm 11.04$ & $\mathrm{~F}=0.44, \mathrm{p}=0.64$ \\
\hline
\end{tabular}

Table 3: Comparison of microalbumin by level of HbA1c among nephropathy patients

\begin{tabular}{|c|c|c|c|c|}
\hline $\begin{array}{c}\text { HbA1c } \\
\%\end{array}$ & $\begin{array}{c}\text { No. of } \\
\text { patients }\end{array}$ & $\begin{array}{c}\text { No. with } \\
\text { nephropathy }\end{array}$ & $\begin{array}{c}\text { \% with } \\
\text { nephropathy }\end{array}$ & $\begin{array}{c}\text { Urine } \\
\text { microalbumin } \\
\text { among } \\
\text { nephropathy }\end{array}$ \\
\hline$<7$ & 42 & 5 & 11.9 & $132.80 \pm 90.33^{1}$ \\
\hline $7-8.9$ & 158 & 83 & 52.5 & $159.89 \pm 70.16^{1}$ \\
\hline $9-10.9$ & 50 & 42 & 84.0 & $246.35 \pm 126.29^{1}$ \\
\hline F=12.04, $\mathrm{p}=0.001, \mathrm{p}=0.0001$ (Multiple comparison tests) \\
\hline
\end{tabular}

The urine microalbumin level was also significantly $(\mathrm{p}=0.001)$ higher among the patients of nephropathy of HbA1c

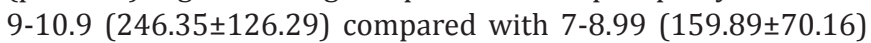
and $<7$ (132.80 \pm 90.33$)$ (Table 3).

\section{Discussion}

Persistent Hyperglycemia beyond the limit of renal thresh hold leads development of diabetic kidney diseases and HbA1c variability. .[11] In our study , HbA1c were significantly higher among nephropathy than non-nephropathy and increased with diabetes duration although all receiving oral hypoglycemic agents (OHA) ,similar finding in previous studies.[12-15] Moreover, significant differences were observed in duration of diabetes cut -off between $<5$ and 5-10 ( $\mathrm{p}=0.04),<5$ and $>10$ (years) among nephropathy, similar association were observed diabetes duration and higher HbA1C values in recent studies.[16-17] Variation may be sensitivity of HbA1c for detecting glycemic variability,18 cellular metabolic memory.[18-20] Predominantly, signal of previous poor glycemic control manifests development of microvasular complication.[21-22] Therefore, HbA1c cut-off variation for nephropathy associated with duration of diabetes due to glucose fluctuations might enhances oxidative stress.[23]

In present study, comparison of lipid levels among nephropathy patients observed that TG significantly altered with increased level of glycosylation which is accordance in previous long term indian observation study.[24]

Dyslipidemia is one of the common conditions associated with a poor glycemic control in type -2 DM. The pathogenesis of dyslipidemia in type -2 DM is a decrease in activity of lipoprotein lipase due to insulin deficiency or resistance. Under the action of insulin, enzyme lipoprotein lipase metabolizes lipids in a healthy individual. In type $2 \mathrm{DM}$, the relative insulin deficiency and decreased adiponectin causes decrease lipoprotein lipase activity resulting in high LDL-c, TG and low HDL-c. Qualitative defects in LDL are also seen in type 2 diabetes including atherogenic, glycated or oxidized LDL further amplifying the risk of Atherogenesis. [25-26] Lebovitz et. Al mentioned the lipotoxic mechanism by triglyceride which interferes with gastric or neural pathway which regulates glycemic control.[27] In another study ,positive HbA1c correlation with triglycerides as prognostic indicator for the target organ damage.[28]

Increased urine microalbumin was found among HbA1c 9-10.9 and high prevalence of nephropathy observed between HbA1c 9-10.9 and 7-8.99 compared to HbA1c <7.00. Similar results reported by Parving et al. [29] observed that HbA1c cut -off of 7.5\%, microalbuminuria (39\%) and overt albuminuria in $9.8 \%$ of the patients while other remained normoalbuminuric. Cummings et.al.[30] suggested that Hba1c cut-off should be $<7 \%$ for renal protection. In light of our interpretation, $\mathrm{HbA} 1 \mathrm{c}$ range $7.0-7.5 \%$ considered as good glycemic control to retard long term damage .

\section{Conclusion}

Recently global norms for glycemic control by American college of physician target HbA1c level between 7-8\% in most patients with type-2 diabetes as against the traditional 6.5$7 \%$, which has been followed over decades. Recommendation is leading conflict among the diabetologist, nephrologist to achieve target blood glucose level for management of diabetes complication. Therefore, Indian Asian ethnicity, race ,age 
,diabetes duration , diet and drug choice are the major dependent factor for determining $\mathrm{HbA1c}$ target. In our research concluded that vascular complication was more prominent in long duration of diabetes and HbA1c variability observed among nephropathy group and high prevalence of nephropathy in type -2 diabetes patients receiving fixed oral hypoglycemic drugs along with ACE inhibitors regimen since known diabetic duration.

\section{References}

1. Lee J, Son H, Ryu OH: Management status of cardiovascular disease risk factors for dyslipidemia among Korean adults. Yonsei Med J. 2017;58(2):326-338. Doi: 10.3349/ymj.2017.58.2.326

2. Dake AW, Sora ND: Diabetic dyslipidemia review: an update on current concepts and management guidelines of diabetic dyslipidemia. Am J Med Sci. 2016;351(4):361-365. Doi: 10.1016/j.amjms.2016.01.020

3. LeeSY.and Choi ME. Urinary biomarkers for early diabetic nephropathy: beyond albuminuria. Pediatr. Nephrol. 2015;30(7):1063-1075. Doi: 10.1007/s00467-014-2888-2

4. Couser, WG, Remuzzi G, Mendis S. and Tonelli M. The contribution of chronic kidney disease to the global burden of major noncommunicable diseases. Kidney Int. 2011;80(12):1258-1270. Doi: 10.1038/ ki.2011.368

5. Currie G, McKay G. and Delles C. Biomarkers in diabetic nephropathy: Present and future. World J Diabetes. 2014;5(6):763-776. Doi: 10.4239/wjd.v5.i6.763

6. American Diabetes Association . Standards of medical care in diabetes - 2014. Diabetes Care. 2014;37(Suppl 1):S14-S80. Doi: 10.2337/ dc14-S014

7. Cavalot F. Do data in the literature indicate that glycaemic variability is a clinical problem? Glycaemic variability and vascular complications of diabetes. Diabetes Obes Metab. 2013;15(Suppl 2):S3-S8. Doi 10.1111/dom.12140

8. Kilpatrick ES, Rigby AS, Atkin SL. A1C variability and the risk of Microvascular complications in type 1 diabetes: data from the Diabetes Control and Complications Trial. Diabetes Care. 2008;31(11):21982202. Doi: $10.2337 / \mathrm{dc} 08-0864$

9. Sugawara A, Kawai K, Motohashi S, Saito K, Kodama S, Yachi Y, et al. $\mathrm{HbA(1c)}$ variability and the development of microalbuminuria in type 2 diabetes: Tsukuba Kawai Diabetes Registry 2. Diabetologia 2012;55(8):2128-2131. Doi: 10.1007/s00125-012-2572-7

10. Vijay Vishwanathan, C. Snehalata, Terin mathai, Muthu Jayaraman, A. Ramachandran - Cardiovascular Morbidity in Proteinuric South Indian NIDDM Patients. Diabeties Research and Clinical Practice. 1998;39(1):63-67.

11. Agarwal R, Light RP. Relationship between glycosylated hemoglobin and blood glucose during progression of chronic kidney disease. Am J Nephrol 2011;34(1):32-41. Doi: 10.1159/000328737

12. Ghosh S, Lyaruu I, Yeates K. Prevalence and factors associated with microalbuminuria in type 2 diabetic patients at a diabetes clinic in Northern Tanzania. Afr J Diabetes Med. 2012;20:43-46.

13. Thakkar B, Arora K, Vekariya R, Lulania M, Agnihotri AS. Prevalence of microalbuminuria in newly diagnosed type 2 diabetes mellitus. NJIRM 2011;2(4):22-25
14.DadhaniaBP, AravatAH, DhruvaGA. Study of microalbuminurea in diabetes type 2 patients as marker of morbidity. Diabetologia. 2012;37:867-75.

15. Hsu CC, Chang HY, Huang MC, Hwang SJ, Yang YC, Lee YS, et al. HbA1c variability is associated with microalbuminuria development in type 2 diabetes: A 7-year prospective cohort study. Diabetologia 2012;55(12):3163-3172. Doi: 10.1007/s00125-012-2700-4

16. Ko S, Park S, Cho J, Shin K, Lee S, et al. Influence of the duration of diabetes on the outcome of a diabetes self-management education program. Diabetes Metab J. 2012;36(3):222-229.

17.Viswanathan V, Tilak P, Kumpatla S. Risk factors associated with the development of overt nephropathy in type 2 diabetes patients: A 12 years observational study. Indian J Med Res. 2012;136(1):46-53.

18. Kilpatrick ES. The rise and fall of $\mathrm{HbA}(1 \mathrm{c})$ as a risk marker for diabetes complications. Di- abetologia. 2012;55(8):2089-2091. Doi: 10.1007/ s00125-012-2610-5

19. Schisano B, Tripathi G, McGee K, McTernan PG, Ceriello A. Glucose oscillations, more than constant high glucose, induce p53 activation and a metabolic memory in human endothelial cells. Diabetologia. 2011;54(5):1219-1226. Doi: 10.1007/s00125-011-2049-0

20. Keating ST, El-Osta A. Glycemic memories and the epigenetic component of diabetic ne- phropathy. Curr Diab Rep 2013;13(4):574581. Doi: 10.1007/s11892-013-0383-y

21. Nathan DM, Cleary PA, Backlund JY, Genuth SM, Lachin JM, et al. Intensive diabetes treatment and cardiovascular disease in patients with type 1 diabetes. N Engl J Med. 2005;353(25):2643-2653. Doi: 10.1056/NEJMoa052187

22. Tonna S, El-Osta A, Cooper ME, Tikellis C. Metabolic memory and diabetic nephropathy: potential role for epigenetic mechanisms. Nat Rev Nephrol. 2010;6(6):332-441. Doi: 10.1038/nrneph.2010.55

23. Brownlee M. The pathobiology of diabetic complications: a unifying mechanism. Diabetes. 2005;54(6):1615-1625.

24.Viswanathan Vijay, Priyanka Tilak \& Satyavani Kumpatla Risk factors associated with the development of overt nephropathy in type 2 diabetes patients: A 12 years observational study Indian J Med Res, 2012;136(1):46-53.

25.Sarfraz M, Sajid S, Ashraf MA. Prevalence and pattern of dyslipidemia in hyperglycemic patients and its associated factors among Pakistani population. Saudi J Biol Sci. 2016;23(6):761-766. Doi: 10.1016/j. sjbs.2016.03.001

26. Verges B. Lipid modification in type 2 diabetes: the role of LDL and HDL . Fundam Clin Pharmacol. 2009;23(6):681-685. Doi: 10.1111/j.14728206.2009.00739.x

27. Lebovitz HE, Ludvik B, Yaniv I, et al. Fasting plasma triglycerides predict the glycaemic response to treatment of type 2 diabetes by gastric electrical stimulation. A novel lipotoxicity paradigm. Diabetic Med. 2013;30(6):687-693. Doi: 10.1111/dme.12132

28. Hussain A, Ali I, Ijaz M, Rahim A. Correlation between hemoglobin A1c and serum lipid profile in Afghani patients with type 2 diabetes: hemoglobin A1c prognosticates dyslipidemia. Ther Adv Endocrinol Metab. 2017;8(4):51-57. Doi: 10.1177/2042018817692296 
29. Parving H, Lewis J, Ravid M, Remuzzi G, Hunsicker L. The DEMAND investigators. Prevalence and risk factors for microalbuminuria in a referred cohort of type II diabetic patients: A global perspective, Kidney International. 2006;69(11):2057-2063. Doi: 10.1038/ sj.ki. 5000377
30. Cummings DM, Larsen LC, Doherty L, Lea CS, Holbert D. Glycemic control patterns and kidney disease progression among primary care patients with diabetes mellitus. J Am Board Fam Med. 2011;24(4):391398. Doi: 10.3122/jabfm.2011.04.100186 